\title{
Genetics in inborn errors of immunity: pediatric autoinflammatory phenotypes and the underlying genetic causes in 125 families
}

Yvonne Poker

Sandra von Hardenberg ( $\nabla$ vonhardenberg.sandra@mh-hannover.de )

Insitut of human genetics https://orcid.org/0000-0002-3680-7450

Winfried Hofmann

Ming Tang

Ulrich Baumann

Nicolaus Schwerk

Martin Wetzke

Viola Lindenthal

Bernd Auber

Brigitte Schlegelberger

Hagen Ott

Philipp von Bismarck

Dorothee Viemann

Frank Dressler

Christian Klemann

Anke Katharina Bergmann

\section{Research Article}

Keywords: Autoinflammatory diseases, familial Mediterranean fever, whole exome sequencing, genetic diagnostics, inborn errors of immunity

Posted Date: February 1st, 2022

DOI: https://doi.org/10.21203/rs.3.rs-1296021/v1

License: (c) (i) This work is licensed under a Creative Commons Attribution 4.0 International License.

Read Full License 
Genetics in inborn errors of immunity: pediatric autoinflammatory phenotypes and the underlying genetic causes in 125 families

Yvonne Poker $^{1 *}$ Sandra von Hardenberg ${ }^{1 * \S}$, Winfried Hofman ${ }^{1}$, Ming Tang ${ }^{1,7}$, Ulrich Baumann ${ }^{2}$, Nicolaus Schwerk ${ }^{2}$, Martin Wetzke ${ }^{2}$, Viola Lindenthal ${ }^{3}$, Bernd Auber ${ }^{1}$, Brigitte Schlegelberger ${ }^{1}$, Hagen Ott ${ }^{4}$, Philipp von Bismarck ${ }^{5}$, Dorothee Viemann ${ }^{2,6}$, Frank Dressler ${ }^{2}$, Christian Klemann ${ }^{2}$, Anke Katharina Bergmann $^{1}$

${ }^{1}$ Department of Human Genetics, Hannover Medical School, 30625 Hannover, Germany; ${ }^{2}$ Department of Pediatric Pneumology, Allergology and Neonatology, Hannover Medical School, 30625 Hannover, Germany; ${ }^{3}$ Department of Pediatrics and Pediatric Hematology/Oncology, University Children's Hospital, Klinikum Oldenburg AöR, 26133 Oldenburg, Germany; ${ }^{4}$ Division of Pediatric Dermatology, Children's Hospital Auf der Bult, Hannover, Germany; ${ }^{5}$ Department of Pediatrics, University Medical Center Schleswig-Holstein, Campus Kiel, 24105 Kiel, Germany; ${ }^{6}$ Translational Pediatrics, Department of Pediatrics, University Hospital Würzburg, Germany; ${ }^{7}$ L3S Research Center, Leibniz University Hannover, Germany

*contributed equally

Running title: Genetics of pediatric autoinflammatory diseases

We do not have any conflicts of interest.

$\S$ Corresponding author:

Sandra v. Hardenberg

Department of Human genetics

Hannover Medical School (MHH)

OE 6300, Carl-Neuberg-Str. 1

30625 Hanover, Germany

Phone: +49 511 532- 83516

Fax: +49 511 532-161064

Email: vonhardenberg.sandra@mh-hannover.de

ORCID: 0000-0002-3680-7450 


\begin{abstract}
Monogenic autoinflammatory diseases (AID) encompass a growing group of inborn errors in the innate immune system causing unprovoked or exaggerated systemic inflammation. Diagnosis of monogenic AID requires an accurate description of the patients' phenotype and the identification of highly penetrant genetic variants in single genes is pivotal. In a routine genetic diagnostic setting, we performed whole exome sequencing (WES) of 125 pediatric patients with suspected monogenic AID. Datasets were analyzed in a step-wise approach to identify the most feasible diagnostic strategy: First, we analyzed a virtual gene panel including 13 genes associated with known AID and, if no genetic diagnosis could be established, followed by the analysis of a virtual panel including 420 genes published by the International Union of Immunological Societies associated with all known inborn error of immunity (IEI). Subsequently WES data were analyzed without pre-filtering for known AID/IEI genes. Analyzing 13 genes yielded a definite diagnosis in $16.0 \%(n=20)$. The diagnostic yield was increased by analyzing 420 genes to $21.8 \%(n=26)$. Importantly, expanding the analysis to WES data did not increase the diagnostic yield in our cohort, neither in single WES analysis nor in trio-WES analysis. The study highlights that analyzing virtual gene panels based on WES that include the majority of known genes causing AID or differential diagnosis can rapidly confirm the diagnosis for a large number of pediatric patients. WES data or trio-WES data analysis as a first-tier diagnostic analysis in patients with suspected monogenic AID is of minor use.
\end{abstract}

Keywords: Autoinflammatory diseases, familial Mediterranean fever, whole exome sequencing, genetic diagnostics, inborn errors of immunity 


\section{Introduction}

Autoinflammatory diseases (AID) are caused by dysregulation or defects of the innate immune system and present with heterogeneous symptoms characterized by recurrent episodes of microbially unprovoked inflammation [1, 2]. This inflammation can be caused by genetic alterations in immuneregulative genes. AID are therefore considered as Inborn Errors of Immunity (IEI), which are categorized by the International Union of Immunological Societies (IUIS) into 10 subgroups with distinctive features [3]. However, due to the heterogeneous clinical presentation of AID, diseases belonging to other IEl subtypes should be taken into consideration as differential diagnosis for AID. Next-generation-sequencing is a powerful diagnostic tool in this process. With the study at hand we aim to support the most feasible diagnostic strategy for patients with suspicion of AID in terms of genetic diagnostics.

The most common AID caused by monogenic alterations are familial Mediterranean fever (FMF), cryopyrin-associated periodic syndromes (CAPS), TNF receptor-associated periodic syndrome (TRAPS), deficiency of IL-1 receptor antagonist (DIRA), and hyper-IgD syndrome (HIDS) [4]. In contrast, PFAPA (periodic fever, aphthous stomatitis, pharyngitis and cervical adenitis) and unclassified SAID (systemic autoinflammatory diseases) are groups without known monogenic etiopathology [5-7].

Clinically, autoinflammation can present with fever, arthritis, mucositis, serositis, musculoskeletal and nervous system involvement as well as skin symptoms (e. g. urticaria, vasculitis, dermatitis). As the phenotypic presentation varies, clinical consensus criteria for initiating genetic testing are not clearly defined. A genetically confirmed diagnosis enables tailored treatment, prognostic estimation of disease process and familial risk estimates. On the other hand, exclusion of an underlying monogenic cause in certain cases, e.g. unusual PFAPA cases, also allows to optimize clinical classification and empirical treatment.

Still over half of all patients presenting with AID do not undergo genetic diagnostics $[8,9]$. Initiating genetic testing in AID usually depends on the treating physician's evaluation, experience and general understanding of genetics. In addition, the decision whether to test raises ethical and psychosocial issues (e.g. cost), and it can be complex to interpret the results. Apart from this, informing patients of their genetic status and helping patients and families deal with the consequences of a genetic diagnosis is challenging in physicians' daily routine [10]. Therefore, it is essential to identify those patients who should be prioritized.

The Mediterranean fever gene MEFV, which codes for pyrin, was first sequenced in 1997 [11, 12]. Since then, according to the IUIS and Infevers database, around 40 other genes have so far been described to cause monogenic AID with a broad range of clinical presentations $[3,13]$. In general, the genetic causes of AID mostly remain unclear; it is already obvious, however, that genetic testing of only a small 
set of genes is insufficient for achieving optimal genetic support and increasing the diagnostic rate in AID. Due to technological advancements and growing experience in genetic diagnostics based on whole exome/whole genome (WES/WGS) sequencing, analyzing WES/WGS data instead of gene panels has become a diagnostic routine in many diagnostic laboratories. Moreover, WES/WGS data can be reevaluated, and availability of new clinical information could lead to more conclusive genetic diagnosis, as shown in other disease entities [14]. To decipher clinical relevance, phenotypic correlation and optimal comprehension of genetic testing in AID, we conducted a step-by-step analysis of 125 unrelated children with a clinical presentation of AID. We applied analyzing different virtual gene panels based on WES as well as complete WES or WGS data analysis to the patients and correlated these with the clinical and phenotypic presentation.

\section{Methods and Materials}

\section{Patient cohort}

The study was conducted in accordance with the general principles outlined in the Declaration of Helsinki [15] and approved by local Institutional Review Board of Hanover Medical School (ID 9849_BO_K_2021).

The analyzed cohort includes 125 index patients diagnosed with AID who were transferred to us for genetic testing between 2018 and 2021. In order for the patients to be included in the study, the following criteria had to be met: evidence of systemic inflammation, a likely monogenic disease, and suspected causality with a particular gene [16].

\section{Sample preparation, whole-exome sequencing and data analysis}

WES was performed in all 125 enrolled patients, as well as in 36 parents as part of trio-WES. Sample preparation, sequencing, and data analysis are described in supplemental data.

\section{Array CGH}

Array-based comparative genomic hybridization (arrayCGH) was performed as published elsewhere [17]. Results are reported according to the International System for Human Cytogenomic Nomenclature 2020 (ISCN 2020).

\section{WES analysis}

First, WES data of all patients were screened with virtual gene panel. For the virtual gene panel 1 (vPANEL_1), we chose 13 genes where pathogenic variants had been reported to be causative for an AID, namely IL1RN, IL36RN, LPIN2, MEFV, MVK, NLRC4, NLRP12, NLRP3, NOD2, PLCG2, PSMB8, PSTPIP1 and TNFRSF1A. Patients without a definitive diagnosis after screening of the aforementioned genes 
were screened using a second virtual gene panel (vPANEL_2), including 420 genes published by the IUIS Expert Committee as causative for IEI [10, 18]. All analyzed genes are listed in Supplemental Table S2. Further information about (e.g. filter strategies) can be found in supplemental data.

\section{Blood parameters for inflammatory markers}

The treating physicians provided clinical and laboratory data. Blood parameters for inflammatory markers were compiled retrospectively for each patient, preferably before initiation of therapy. Levels of CRP (C-reactive protein) ( $\mathrm{mg} / \mathrm{l}$ ) and ESR (erythrocyte sedimentation rate) $(\mathrm{mm} / \mathrm{h}$ ) were documented, as well as serum calprotectin $(\mathrm{ng} / \mathrm{ml})$, serum amyloid $A(\mathrm{mg} / \mathrm{l})$, and blood count (Supplemental Table S3).

\section{Results}

\section{Clinical phenotypes}

The mean age of the patients at genetic testing was 6.8 years ( 4 days - 18 years). The total cohort consisted of $60(48 \%)$ female and $65(52 \%)$ male patients. Ancestry were self-reported as Northern European ( $n=58)$, Mediterranean ( $n=33)$, and Middle Eastern $(n=25)$, as well as African $(n=2)$, Asian $(n=2)$, Latin American $(n=1)$, assorted ethnicity (Northern European and Mediterranean; $n=3$ ), and unknown ( $n=1)$. In 12 families (9.6\%), consanguinity (first- or second-degree cousins) was selfreported. Detailed patient characteristics are listed in Supplemental Table S1.

The median age of onset of the patients was 3.8 years ( 0.1 to 16.7 years). On average, the latency between disease manifestation and genetic workup was three years ( 0.1 to 14.5 years). Hospitalization (at least once) was required in 61 patients (48.8\%) due to their underlying inflammatory disease. Seven patients (5.6\%) needed ICU treatment, including two with a fatal outcome (patients ID23 and ID48).

To define the patient phenotypes according to the Human Phenotype Ontology (HPO) [18], we classified the symptoms of AID presentation according to the 15 main functional systems as follows: body temperature regulation, gastrointestinal tract (GI tract), musculoskeletal system, skin barrier, mucous membranes, visual system, effusion, lymphatic system, susceptibility to infection, respiratory system, cardiovascular system, kidney and urinary tract, auditory system, nervous system, and laboratory biomarkers positive for systemic inflammation.

Among the 15 main functional systems according to HPO, the top six functional system phenotypes we found in our cohort were: 1 . body temperature regulation $(n=92,73.6 \%), 2$. positive laboratory biomarkers ( $n=86,68.8 \%), 3$. Gl tract $(n=54,43.2 \%)$, 4. musculoskeletal system ( $n=40,32 \%), 5$. lymphatic system and skin barrier, each reported in 35 patients (28\%) (Supplemental Figure S4). 


\section{Results through genetic workup}

Patients were screened step-by-step for VPANEL_1, VPANEL_2 or WES_3 (see Material and Methods section and Figure 1) to determine to what extent genetic testing was needed. Detailed information about the genetic workup of each patient is provided in supplemental Table S5. Applying testing with VPANEL_1, pathogenic variants (PV) or likely pathogenic variants (LPV) were identified in 20/125 individuals $(16.0 \%)$ in the genes MEFV $(n=19)$ and NLRP3 $(n=1)$ (Table 1). 25 variants of unknown significance (VUS, class 3) were identified in 30/125 patients (24\%) (Supplemental Table S6). Applying testing with VPANEL_2, PVs or LPVs were identified in 6/125 individuals (4.8\%) in ADA2 (n=1), CYBB $(n=1), \operatorname{IL1ORB}(n=1), \operatorname{MS} 4 A 1(n=1), \operatorname{RIPK1}(n=1)$ and TTC37 (n=1). 11 VUS were identified in 11/125 patients (11.2\%) (Supplemental Table S6). Overall, monoallelic LPV were identified in four genes which are known to follow an autosomal recessive inheritance (CIITA, NCKAP1L, MVK, POLE2), but a second variant was not detected (Supplemental Table S6). Likewise, analyzing CNVs in these regions yielded no segmental gains or losses in DNA sequence. VUS should not be used in clinical decision-making yet, but monitoring of the patient for the disorder in question was recommended.

For the remaining 99 patients, WES data (WES_3) were filtered to detect the most likely deleterious variants highly suspicious for AID. Additionally, HPO terms were used according to the patient's phenotype. A brief literature review was performed on each gene to evaluate clinical significance and its role in IEI, especially in AID and the inflammasome. 15 VUS, highly suspicious for AID, were identified in 14 genes in 13 patients (ALPK1, AXL, BAHD1, CARD10, IGF2R, IRGM, LTK, MAPK14, NFKBIL1, PLA2R1, RHBDF1, RNF220, TRIM21, and ZC3H12A) (Supplemental Table S7). Some of the identified genes (e.g. ALPK1, IRGM and NFKBIL1) are linked to a specific phenotype in OMIM [19]. VUS not fulfilling the aforementioned criteria are not listed and need further evaluation.

Because of the advantage of incorporating inheritance information, trio-WES was initially requested in 17 patients (13.2\%) to achieve a fast genetic result in critically ill children. Segregation analysis to evaluate highly suspicious variants found in singleton WES was performed using Sanger sequencing $(n=14)$ and WES $(n=1)$. Array CGH was performed in another 5 patients because of familial mental retardation or CNV alterations identified through WES; no pathogenic CNV were identified. In order to exclude an underlying second genetic disease in the patients who had already received a genetic diagnosis through VPANEL_1 or VPANEL_2, these patients were finally also examined using WES_3. None of these further evaluations identified a clear PV.

Below, we describe the six patients with positive genetic results after VPANEL_2 screening.

Patient ID23 suffered from severe pulmonary arterial hypertension with right heart failure due to diaphragmatic herniation resulting in lung hypoplasia. He underwent sequential double lung 
transplantation at age 5 years followed by a complicated course with multiple suspected acute rejections and chronic allograft dysfunction. As the patient showed signs of autoinflammatory symptoms whenever steroids were reduced, genetic testing was initiated. This identified the previously unreported hemizygous LPV c.1511G>C p.(Gly504Ala) in the CYBB gene. PVs of CYBB are responsible for the most common form of chronic granulomatous disease [20-22] (CGD, MIM306400), a primary immunodeficiency in which phagocytic cells display little or no NADPH oxidase activity [23, 24]. Flow cytometry assessing respiratory burst activity in phagocytes confirmed the CDG diagnosis. Based on the IUIS classification of immunological diseases, this disease belongs to the "Congenital Defects of Phagocyte Number or Function" (Table 5) or "Defects in Intrinsic and Innate Immunity" [25] (Table 6). In addition, using the WES_3 data analysis, we also identified a second LPV c.1276delG p.(Ala426Profs*45) in the ZFPM2 gene. This variant has not been previously reported. Heterozygous PVs in ZFPM2 cause congenital diaphragmatic hernias and structural anomalies of the diaphragm [26]. A reduced penetrance is recorded [27]. He finally passed away at age of 8 years due to terminal allograft dysfunction.

In patient ID26, two compound heterozygous LPVs were detected in the TTC37 gene (c.2453_2454delTG p.(Val818Glufs*5) and c.(-228+1216_-228+1222)_(326+92_326+98)del p.?), leading to a diagnosis of tricho-hepato-enteric syndrome $1[34,35]$. The patient experienced the first symptoms at the age of 6 months; these included severe pneumonia followed by recurrent infection and persistent diarrhea in the following years. This patient nevertheless showed a mild phenotype with spontaneous remission of diarrhea, no hypogammaglobulinemia, and low antibody production after vaccination against pneumococcus. The IUIS classifies the underlying disease as "Combined Immunodeficiency with Associated or Syndromic Features" [25] (Table 2b).

In patient ID18, the identification of the homozygous PV c.477G>A p.(Trp159*) in the IL1ORB gene confirmed the diagnosis of an IL-10-receptor defect [28]. The variant has been previously described in 6 other patients [28]. The parents of this patient are first cousins. The patient showed the first symptoms of autoinflammatory disease during the neonatal period. Symptoms included failure to thrive, recurrent episodes of fever, maculopapular skin lesions, intermittent bloody diarrhea, and hearing impairment. Allogeneic bone marrow transplantation led to remission. Based on the IUIS classification of immunological diseases, this disease belongs to "Diseases of Immune Dysregulation" [25] (Table 4).

In patient ID55, a heterozygous LPV was detected in the gene MS4A1 (c.203dup p.(Lys9Valfs*14)). Biallelic PVs in MS4A1 are known to cause CVID with hypogammaglobulinemia (MIM613495). Heterozygous carriers have been described to have impaired expression of CD20 on IgG4-expressing plasma cells [29]. Segregation analysis revealed that the healthy father as well as the deceased 
grandmother, who suffered from M. Ormond, were carriers as well. A variable penetrance can thus be assumed. Based on the IUIS classification of immunological diseases, this disease belongs to "Predominantly Antibody Deficiencies" [25] (Table 3).

Patient ID19 presented with classical symptoms of a periodic fever syndrome like limb pain, splenomegaly, and swollen joints with additional effusion later during the course of illness. Genetic testing identified a homozygous PV in the gene ADA2 (c.139G>A p.(Gly47Arg)). Biallelic PVs in ADA2 led to vasculitis with autoinflammation (MIM61588), explaining the phenotype observed in our patient $[30,31]$.

In patient ID25, WES identified a heterozygous PV in the gene RIPK1 (c.970G>C p.(Asp324His)) as de novo. This variant was already described to cause cleavage-resistant RIPK1-induced autoinflammatory syndrome (CRIA-syndrome) [32, 33]. Our patient showed recurrent fever without a certain periodicity, hepatosplenomegaly, lymphadenitis, and microcytic anemia. Treatment with IL-6-inhibitors improved the symptoms.

\section{Expanding differential diagnosis through genetic testing in suspected AID}

We initially sequenced all patients in our cohort with a suspicion of an AID. In some patients, however, the genetic results and following clinical reassessment confirmed a final clinical diagnosis that belongs to a subgroup of IEl other than AID. Therefore, we categorized the patients' final clinical diagnosis into the following subclasses: genetic autoinflammation (AID) $(n=22)$, patients with transient symptoms ( $n=16)$, juvenile idiopathic arthritis (JIA) including systemic JIA (SJIA) $(n=14)$, PFAPA $(n=16)$, unclassified SAID ( $n=33)$, Morbus Behçet $(n=3)$, defined disease of the immune system without known genetic cause $(n=17)$, and IEI_other than autoinflammation (IEI) $(n=4)$.

AID was subdivided into FMF ( $\mathrm{n}=19)$, VAIHS-syndrome (ADA2; $\mathrm{n}=1)$, CAPS (NLRP3; $\mathrm{n}=1)$ and CRIAsyndrome (RIPK1; $\mathrm{n}=1)$. IEl other than AID was subdivided into Inflammatory bowel disease 25 (IL1ORB; $\mathrm{n}=1)$, chronic granulomatous disease $($ CYBB; $\mathrm{n}=1)$, tricho-hepato-enteric syndrome $1(\operatorname{TTC} 37 ; \mathrm{n}=1)$ and CVID with hypogammaglobulinemia (MS4A1; $\mathrm{n}=1$ ) (Figure 2).

\section{Patients with PVs in the MEFV gene}

FMF is an autosomal recessive disorder characterized by recurrent, self-limited, sporadic attacks of fever and serosal inflammation [34]. Sequencing of MEFV is the gold standard for suspected cases of AID. Over 370 MEFV sequence variants have so far been identified in FMF patients, which occur mostly in the gene's 2 nd and 10th exons [11]. In our cohort, 19/125 patients carried at least one PV in the MEFV gene (Table 1).

Biallelic PVs in the MEFV gene confirm the clinical diagnosis of FMF, although heterozygous individuals have also been shown to exhibit classical disease-related symptoms. As demonstrated in Figure 3, 
laboratory biomarkers showed signs of inflammation in $100 \%(n=9)$ of the patients carrying biallelic PVs and $62 \%(n=5)$ of the patients carrying monoallelic PVs. Differences in other affected functional systems between biallelic and monoallelic carriers of MEFV PVs are shown in Figure 3.

Blood levels of serum calprotectin, CRP, ESR, and serum amyloid A may be of value to monitor disease course and the patient's response to treatment [35]. The differences became obvious when we compared these laboratory biomarkers of patients with biallelic PVs in MEFV with those with monoallelic PVs in MEFV. Median serum calprotectin was $55240 \mathrm{ng} / \mathrm{ml}$ in the group of biallelic carriers $(n=9)$ and $6960 \mathrm{ng} / \mathrm{ml}$ in the group of monoallelic carriers $(n=9)(p<0.001$, ref value $<2940 \mathrm{ng} / \mathrm{ml})$. Considering serum amyloid A, the median level in the group of biallelic carriers was $130 \mathrm{mg} / \mathrm{l}$, whereas the median level in the group of monoallelic carriers was $3.7 \mathrm{mg} / \mathrm{l}(p=0.07$, ref value $6.4 \mathrm{ng} / \mathrm{ml}$ ) (Figure 4, Supplemental Table S3).

\section{Discussion}

A monogenic AID diagnosis is based on a careful interpretation of the clinical phenotype and results of molecular genetic analysis. We performed genetic testing in a cohort of 125 pediatric patients with suspected AID and addressed the use of different virtual panels (13 AID gene VPANEL_1/ 420 IUIS gene VPANEL_2) and WES data (WES_3) analysis. In the first approach, 125 patients were analyzed by VPANEL_1, which yielded a clear pathogenic variant (PV) in 20 patients (16.0\%). Analyzing the remaining 104 patients by VPANEL_2 led to an additional positive genetic result in 6 patients (4.8\%). Remarkably, in 3 of these 26 patients, an IEI different to AID was diagnosed based on genetic results (IL1ORB defect, chronic granulomatous disease, and tricho-hepato-enteric syndrome). In our cohort, WES data analysis (WES_3) did not establish a reliable diagnosis of the inflammatory phenotype. Furthermore, trio-WES in 18 patients of our cohort did not increase our diagnostic yield.

In summary, the overall diagnostic yield in our cohort was $20.8 \%$. In addition, we identified in $43.2 \%$ VUS, which could become clinically relevant in the future. This rate supports other studies focusing on the genetic diagnosis of $\operatorname{AID}[5,13,36-41]$. Cohorts of equal size $[5,13,36]$ and larger cohorts [41] were both evaluated in these studies, and some only included pediatric patients [12, 39, 42, 43], while others also included adults [36-38, 41, 44, 45]. A higher positive yield was reported in studies with a more selective choice in the patient cohort, e.g. a diagnostic yield of $36 \%$ was observed in cohorts with a high rate of consanguinity [43]. Studies focusing on unclassified SAIDs usually show low diagnostic rates as published by Demir et al. [39]. The term "unclassified SAIDs" has been used to describe patients who do not meet the clinical diagnostic criteria for an AID or have clinical findings for more than one AID. There are no diagnostic criteria for these patients $[5,12,46]$ who may have a combination of multiple low-penetrance genetic and environmental factors. In our study, we ranked 30 patients, for whom a definitive diagnosis of a monogenic disease could not be made, in the 
"unclassified SAIDs" category. Among these, 8 patients had VUS in LPIN2, TNFAIP3, MEFV, NLRC4, NLRP1 or NLRP3, which may contribute to their phenotype.

Confirmatory testing for phasing variants in recessive disorders and the determination of inheritance using sanger sequencing led to an upgrading (ID20, ID22, ID23, ID25) or downgrading (ID39, ID51, ID55, ID59, ID68, ID77, ID107, ID109) of their pathogenicity classification. Systematic segregation analyses have not been included in recent reports. Moreover, in contrast to other studies [5, 37, 38, 41, 43-45], our study included 18 trio-WES. Trio-WES was traditionally considered superior for genetic disease diagnosis and the determination of de novo occurrence often upgraded the pathogenicity classification of variants. Furthermore trio-WES normally decreases turnaround time for positive and negative cases [47], therefore yielding a quicker diagnosis with a synopsis of parental and offspring genetic data.

The average of the diagnostic yields reported in several studies focusing on trio-WES in children with any kind of suspected genetic disease and collected by a literature review of Dragojlovic et al was $34.4 \%(\mathrm{SD}=5.9 \%, n=13)$ for trio-WES and $26.6 \%(\mathrm{SD}=8.0 \%, n=7)$ for single WES [48]. In contrast, trio-WES did not yield an additional genetic diagnosis in our study. A likely explanation is the difference in our study's experimental design and perhaps its stringent patient selection criteria. Likewise, the occurrence of two PVs or LPVs in a single WES in a recessive disorder fitting to the patient's presentation is often sufficient to merit nomination for confirmatory phasing. Since trio-WES is more than twice as expensive as single WES, we recommend initially performing only single WES with analysis of a certain set of genes (e.g. VPANEL_1 and VPANEL_2) on patients with non-life-threatening AID.

In 16 patients with suspected PFAPA, virtual panel diagnostics were used to exclude a monogenic cause and none of these patients showed a monogenic cause. However, PFAPA syndrome is a clinical diagnosis with different classification criteria [4, 49-53]. Thomas et al. established the following diagnostic criteria for PFAPA: 1) regularly recurring fevers with an onset before 5 years of age without infection; 2) one of the following signs a) aphthous stomatitis b) cervical lymphadenitis, c) pharyngitis; 3) exclusion of cyclic neutropenia; 4) asymptomatic interval between episodes; 5) normal growth and development (4). Since a relevant number of patients with monogenic periodic fever syndromes also meet the diagnostic criteria for PFAPA syndrome [53], failure to diagnose patients with very similar phenotypes could be fatal. Gattorno et al. demonstrated that $83 \%$ of patients with mevalonate kinase deficiency, $57 \%$ of patients with TRAPS, and $8 \%$ of patients with FMF met the criteria for PFAPA syndrome [53]. Hofer et al. postulated that the exclusion of monogenic periodic fever syndromes should be included in a new version of the criteria [54]. A high allele frequency of MEFV variants (2766\%) has been reported previously in PFAPA patients [55]. Therefore, PFAPA syndrome is not unexpected in children with MEFV variants. The underlying pathogenesis of PFAPA syndrome and the 
genotype-phenotype correlation in MEFV variants are both not fully understood. This suggests more underlying factors determining the phenotype in heterozygous carriers of MEFV variants. Patient ID62, for example, presented with symptoms pointing towards PFAPA syndrome like periodic fever and recurrent pharyngitis and was diagnosed with heterozygous MEFVPV. He was successfully treated with prednisolone, supporting the assumption of an underlying PFAPA syndrome. Based on the Thomas criteria [4] and following monogenic disease exclusion, 16 patients (ID63 to ID78) in our cohort could be diagnosed with PFAPA syndrome.

The highest diagnostic rate was observed in the MEFV gene in 19/27 patients with confirmed genetic diagnosis, representing $70 \%$. The diagnosis of FMF is unproblematic in the presence of characteristic symptoms and positive family history, but in early childhood clinical signs of FMF are difficult to distinguish from more common nonspecific symptoms. Demirkaya et al. published a severity score for familial Mediterranean fever (ISSF) based on chronic sequelae, organ dysfunction, organ failure, frequency of attacks, and increases in acute phase reactants [56]; however, the diagnosis of FMF is usually based on a combination of clinical and genetic factors. High allele frequencies of MEFV PVs have been observed in certain ethnicities [42].

To date, clinical consensus criteria for initiating genetic testing in patients with suspected AID are not clearly defined. To reduce diagnostic costs and time, we recommend using stepwise genetic testing in clinically stable patients based on WES data. Many patients were diagnosed by analyzing a small set of genes specific to common AID. Expanding this set of genes to other genes known to cause human errors of immunity was effective in identifying more PVs (IUIS). Explorative single WES analysis could not offer further diagnostic yield in this study. Nevertheless, in a research setting WES and trio-WES analysis will result in the identification of yet undescribed genes $[61,62]$ or VUS, which could become clinically relevant in the future. This is especially true for complex phenotypes where autoinflammation only accounts for part of the symptoms. 


\section{Acknowledgements}

We are grateful to Manuela Losch, Bernd Haermeyer and Tim Rajewski for their technical assistance. The authors thank all patients, their families, and treating physicians who participated in this study.

\section{Funding}

AKB, BA, BS, MT, SH, WH and YB were supported by Cluster of Excellence RESIST (Resolving Infection Sustainability) EXC 2155/1 Deutsche Forschungsgemeinschaft (German Research Foundation)

\section{Competing Interests}

The authors have no relevant financial or non-financial interests to disclose.

\section{Author contributions:}

SH and AKB designed the study; YP, SH, AKB performed genetic data analysis; YP conducted segregation analysis and performed Array-CGH analysis; WH and MT performed bioinformatics analyses; YP, SH, AKB, BS, BA and FD supported data interpretation; FD, CK, VL, UB, NS, MW, BP, DV, HO provided samples and clinical data; YP collected information on family histories; YP, SH and AKB wrote the manuscript.

\section{Data Availability}

The datasets generated during and/or analysed during the current study are available from the corresponding author on reasonable request.

\section{Ethics approval}

This study was performed in line with the principles of the Declaration of Helsinki. Approval was granted by the local Institutional Review Board of Hanover Medical School (ID 9849_BO_K_2021).

\section{Consent to participate}

Written informed consent was obtained from the parents.

\section{Consent to publish}

Written informed consent was obtained from the parents. 


\section{References}

(1) McDermott MF, Aksentijevich I, Galon J, McDermott EM, Ogunkolade BW, Centola M, et al. Germline mutations in the extracellular domains of the 55 kDa TNF receptor, TNFR1, define a family of dominantly inherited autoinflammatory syndromes. Cell 1999 Apr 2;97(1):133-144.

(2) Ben-Chetrit E, Gattorno M, Gul A, Kastner DL, Lachmann HJ, Touitou I, et al. Consensus proposal for taxonomy and definition of the autoinflammatory diseases (AIDs): a Delphi study. Ann Rheum Dis 2018 Nov;77(11):1558-1565.

(3) Tangye SG, Al-Herz W, Bousfiha A, Cunningham-Rundles C, Franco JL, Holland SM, et al. The EverIncreasing Array of Novel Inborn Errors of Immunity: an Interim Update by the IUIS Committee. J Clin Immunol 2021 Apr;41(3):666-679.

(4) Ciccarelli F, De Martinis M, Ginaldi L. An update on autoinflammatory diseases. Curr Med Chem 2014;21(3):261-269.

(5) Thomas KT, Feder HM,Jr, Lawton AR, Edwards KM. Periodic fever syndrome in children. J Pediatr 1999 Jul;135(1):15-21.

(6) Ter Haar NM, Eijkelboom C, Cantarini L, Papa R, Brogan PA, Kone-Paut I, et al. Clinical characteristics and genetic analyses of 187 patients with undefined autoinflammatory diseases. Ann Rheum Dis 2019 Oct;78(10):1405-1411.

(7) Lachmann HJ. Clinical immunology review series: An approach to the patient with a periodic fever syndrome. Clin Exp Immunol 2011 Sep;165(3):301-309.

(8) Papa R, Rusmini M, Volpi S, Caorsi R, Picco P, Grossi A, et al. Next generation sequencing panel in undifferentiated autoinflammatory diseases identifies patients with colchicine-responder recurrent fevers. Rheumatology (Oxford) 2020 Feb 1;59(2):344-360.

(9) Elferink MG, van Zon P, Frenkel J, Harts W, Simon A, van Royen-Kerkhof A, et al. Targeted NGS based hereditary autoinflammatory disorder screening in routine diagnostics, two year experience in the Netherlands. Pediatric Rheumatology 2015;13(1):P51.

10) Pasquier L, Minguet G, Moisdon-Chataigner S, Jarno P, Denizeau P, Volf G, et al. How do nongeneticist physicians deal with genetic tests? A qualitative analysis. European Journal of Human Genetics 2021.

(11) French FMF Consortium. A candidate gene for familial Mediterranean fever. Nat Genet 1997 Sep;17(1):25-31.

(12) International FMF Consortium. Ancient missense mutations in a new member of the RoRet gene family are likely to cause familial Mediterranean fever. The International FMF Consortium. Cell 1997 Aug 22;90(4):797-807.

(13) Infevers. Infevers: an online database for autoinflammatory mutations. Copyright. 2021; Available at: https://infevers.umai-montpellier.fr/. Accessed 10.11., 2021.

(14) Basel-Salmon L, Orenstein N, Markus-Bustani K, Ruhrman-Shahar N, Kilim Y, Magal N, et al. Improved diagnostics by exome sequencing following raw data reevaluation by clinical geneticists involved in the medical care of the individuals tested. Genet Med 2019 Jun;21(6):1443-1451.

(15) World Medical Association. World Medical Association Declaration of Helsinki: ethical principles for medical research involving human subjects. JAMA 2013 Nov 27;310(20):2191-2194. 
(17) Schieck M, Lentes J, Thomay K, Hofmann W, Behrens YL, Hagedorn M, et al. Implementation of RNA sequencing and array CGH in the diagnostic workflow of the AIEOP-BFM ALL 2017 trial on acute lymphoblastic leukemia. Ann Hematol 2020 Apr;99(4):809-818.

(18) Köhler S, Gargano M, Matentzoglu N, Carmody LC, Lewis-Smith D, Vasilevsky NA, et al. The Human Phenotype Ontology in 2021. Nucleic Acids Res 2021 Jan 8;49(D1):D1207-D1217.

(19) OMIM. Online Mendelian Inheritance in Man, OMIM ${ }^{\circledR}$. McKusick-Nathans Institute of Genetic Medicine, Johns Hopkins University (Baltimore, MD). 2021; Available at: https://omim.org/.

(20) Roos D. Chronic granulomatous disease. Br Med Bull 2016 Jun;118(1):50-63.

(21) Bustamante J, Arias AA, Vogt G, Picard C, Galicia LB, Prando C, et al. Germline CYBB mutations that selectively affect macrophages in kindreds with $\mathrm{X}$-linked predisposition to tuberculous mycobacterial disease. Nat Immunol 2011 Mar;12(3):213-221.

(22) Bustamante J, Picard C, Fieschi C, Filipe-Santos O, Feinberg J, Perronne C, et al. A novel X-linked recessive form of Mendelian susceptibility to mycobaterial disease. J Med Genet 2007 Feb;44(2):e65.

(23) Roos D, Kuhns DB, Maddalena A, Roesler J, Lopez JA, Ariga T, et al. Hematologically important mutations: X-linked chronic granulomatous disease (third update). Blood Cells Mol Dis 2010 Oct 15;45(3):246-265.

(24) Winkelstein JA, Marino MC, Johnston RB,Jr, Boyle J, Curnutte J, Gallin JI, et al. Chronic granulomatous disease. Report on a national registry of 368 patients. Medicine (Baltimore) 2000 May;79(3):155-169.

(25) Bousfiha A, Jeddane L, Picard C, Al-Herz W, Ailal F, Chatila T, et al. Human Inborn Errors of Immunity: 2019 Update of the IUIS Phenotypical Classification. J Clin Immunol 2020 Jan;40(1):66-81.

(26) Brady PD, Van Houdt J, Callewaert B, Deprest J, Devriendt K, Vermeesch JR. Exome sequencing identifies ZFPM2 as a cause of familial isolated congenital diaphragmatic hernia and possibly cardiovascular malformations. Eur J Med Genet 2014;57(6):247-252.

(27) Longoni M, Russell MK, High FA, Darvishi K, Maalouf Fl, Kashani A, et al. Prevalence and penetrance of ZFPM 2 mutations and deletions causing congenital diaphragmatic hernia. Clin Genet 2015 Apr;87(4):362-367.

(28) Glocker EO, Kotlarz D, Boztug K, Gertz EM, Schäffer AA, Noyan F, et al. Inflammatory bowel disease and mutations affecting the interleukin-10 receptor. N Engl J Med 2009 Nov 19;361(21):2033-2045.

(29) Grimm KE, Bakke A, O'Malley DP. Abnormal expression of CD20 on IgG4 plasma cells associated with IgG4-related lymphadenopathy. Arch Pathol Lab Med 2013 Sep;137(9):1282-1285.

(30) Lee PY. Vasculopathy, Immunodeficiency, and Bone Marrow Failure: The Intriguing Syndrome Caused by Deficiency of Adenosine Deaminase 2. Front Pediatr 2018 Oct 18;6:282.

(31) Zhou Q, Yang D, Ombrello AK, Zavialov AV, Toro C, Zavialov AV, et al. Early-onset stroke and vasculopathy associated with mutations in ADA2. N Engl J Med 2014 Mar 6;370(10):911-920.

(32) Tao P, Sun J, Wu Z, Wang S, Wang J, Li W, et al. A dominant autoinflammatory disease caused by non-cleavable variants of RIPK1. Nature 2020 Jan;577(7788):109-114.

(33) Lalaoui N, Boyden SE, Oda H, Wood GM, Stone DL, Chau D, et al. Mutations that prevent caspase cleavage of RIPK1 cause autoinflammatory disease. Nature 2020 Jan;577(7788):103-108.

(34) Padeh S, Berkun Y. Familial Mediterranean fever. Curr Opin Rheumatol 2016 Sep;28(5):523-529. 
(35) Berkun Y, Padeh S, Reichman B, Zaks N, Rabinovich E, Lidar M, et al. A single testing of serum amyloid a levels as a tool for diagnosis and treatment dilemmas in familial Mediterranean fever. Semin Arthritis Rheum 2007 Dec;37(3):182-188.

(36) Okano T, Imai K, Naruto T, Okada S, Yamashita M, Yeh TW, et al. Whole-Exome SequencingBased Approach for Germline Mutations in Patients with Inborn Errors of Immunity. J Clin Immunol $2020 \mathrm{Jul} ; 40(5): 729-740$.

(37) Karacan I, Balamir A, Uğurlu S, Aydın AK, Everest E, Zor S, et al. Diagnostic utility of a targeted next-generation sequencing gene panel in the clinical suspicion of systemic autoinflammatory diseases: a multi-center study. Rheumatol Int 2019 May;39(5):911-919.

(38) Batlle-Masó L, Mensa-Vilaró A, Solís-Moruno M, Marquès-Bonet T, Arostegui Jl, Casals F. Genetic diagnosis of autoinflammatory disease patients using clinical exome sequencing. Eur J Med Genet 2020 May;63(5):103920.

(39) Demir F, Doğan Ö, Demirkol YK, Tekkuş KE, Canbek S, Karadağ Ş, et al. Genetic panel screening in patients with clinically unclassified systemic autoinflammatory diseases. Clin Rheumatol 2020 Dec;39(12):3733-3745.

(40) Hoang TK, Albert DA. Novel presentations of periodic fever syndromes: Discrepancies between genetic and clinical diagnoses. Eur J Rheumatol 2019 Jan;6(1):12-18.

(41) Yao Q, Lacbawan F, Li J. Adult autoinflammatory disease frequency and our diagnostic experience in an adult autoinflammatory clinic. Semin Arthritis Rheum 2016;45(5):633-637.

(42) Fathalla BM, Alsarhan A, Afzal S, El Naofal M, Abou Tayoun A. The genomic landscape of pediatric rheumatology disorders in the Middle East. Hum Mutat 2021 Apr;42(4):e1-e14.

(43) Kosukcu C, Taskiran EZ, Batu ED, Sag E, Bilginer Y, Alikasifoglu M, et al. Whole exome sequencing in unclassified autoinflammatory diseases: more monogenic diseases in the pipeline? Rheumatology (Oxford) 2021 Feb 1;60(2):607-616.

(44) Hoang TK, Albert DA. Novel presentations of periodic fever syndromes: Discrepancies between genetic and clinical diagnoses. Eur J Rheumatol 2019 Jan;6(1):12-18.

(45) Omoyinmi E, Standing A, Keylock A, Price-Kuehne F, Melo Gomes S, Rowczenio D, et al. Clinical impact of a targeted next-generation sequencing gene panel for autoinflammation and vasculitis. PLoS One 2017 Jul 27;12(7):e0181874.

(46) Harrison SR, McGonagle D, Nizam S, Jarrett S, van der Hilst J, McDermott MF, et al. Anakinra as a diagnostic challenge and treatment option for systemic autoinflammatory disorders of undefined etiology. JCl Insight 2016 May 5;1(6):e86336.

(47) Kingsmore SF, Cakici JA, Clark MM, Gaughran M, Feddock M, Batalov S, et al. A Randomized, Controlled Trial of the Analytic and Diagnostic Performance of Singleton and Trio, Rapid Genome and Exome Sequencing in III Infants. Am J Hum Genet 2019 Oct 3;105(4):719-733.

(48) Dragojlovic N, Elliott AM, Adam S, van Karnebeek C, Lehman A, Mwenifumbo JC, et al. The cost and diagnostic yield of exome sequencing for children with suspected genetic disorders: a benchmarking study. Genet Med 2018 Sep;20(9):1013-1021.

(49) Marshall GS, Edwards KM, Butler J, Lawton AR. Syndrome of periodic fever, pharyngitis, and aphthous stomatitis. J Pediatr 1987 Jan;110(1):43-46.

(50) Gattorno M, Sormani MP, D'Osualdo A, Pelagatti MA, Caroli F, Federici S, et al. A diagnostic score for molecular analysis of hereditary autoinflammatory syndromes with periodic fever in children.

Arthritis Rheum 2008 Jun;58(6):1823-1832. 
(51) Cochard M, Clet J, Le L, Pillet P, Onrubia X, Guéron T, et al. PFAPA syndrome is not a sporadic disease. Rheumatology (Oxford) 2010 Oct;49(10):1984-1987.

(52) Tasher D, Somekh E, Dalal I. PFAPA syndrome: new clinical aspects disclosed. Arch Dis Child 2006 Dec;91(12):981-984.

(53) Gattorno M, Caorsi R, Meini A, Cattalini M, Federici S, Zulian F, et al. Differentiating PFAPA syndrome from monogenic periodic fevers. Pediatrics 2009 Oct;124(4):721.

(54) Hofer M, Pillet P, Cochard MM, Berg S, Krol P, Kone-Paut I, et al. International periodic fever, aphthous stomatitis, pharyngitis, cervical adenitis syndrome cohort: description of distinct phenotypes in 301 patients. Rheumatology (Oxford) 2014 Jun;53(6):1125-1129.

(55) Celiksoy MH, Ogur G, Yaman E, Abur U, Fazla S, Sancak R, et al. Could familial Mediterranean fever gene mutations be related to PFAPA syndrome? Pediatr Allergy Immunol 2016 Feb;27(1):78-82.

(56) Demirkaya E, Acikel C, Hashkes P, Gattorno M, Gul A, Ozdogan H, et al. Development and initial validation of international severity scoring system for familial Mediterranean fever (ISSF). Ann Rheum Dis 2016 Jun;75(6):1051-1056.

\section{Tables}

Table 1: Pathogenic and likely pathogenic variants identified by VPANEL_1 


\begin{tabular}{|c|c|c|c|c|c|c|c|c|c|c|}
\hline IDPatient & gene & coding DNA (c.) & protein (p.) & coding DNA (c.) & protein (p.) & Transcript & Zygosity & $\begin{array}{c}\text { mode } \\
\text { of } \\
\text { inherit } \\
\text { ance }\end{array}$ & class & Phenotype (MIM) \\
\hline & & \multicolumn{2}{|c|}{ allele 1} & \multicolumn{2}{|c|}{ allele 2} & & & & & \\
\hline 2 & MEFV & c. $2080 A>G$ & p.(Met694Val) & c. $2080 A>G$ & p.(Met694Val) & \multirow{19}{*}{ NM_000243.2 } & hom & $A R, A D$ & 5 & \multirow{19}{*}{$\begin{array}{l}\text { Familial Mediterranean fever, AD } \\
\text { (MIM134610) } \\
\text { Familial Mediterranean fever, AR } \\
\text { (MIM249100) } \\
\text { Neutrophilic dermatosis, acute febrile } \\
\text { (MIM608068) }\end{array}$} \\
\hline 3 & MEFV & c. $2080 A>G$ & p.(Met694Val) & c. $2080 A>G$ & p.(Met694Val) & & hom & $A R, A D$ & 5 & \\
\hline 5 & MEFV & c. $2080 A>G$ & p.(Met694Val) & c. $2080 A>G$ & p.(Met694Val) & & hom & $A R, A D$ & 5 & \\
\hline 6 & MEFV & c. $2080 A>G$ & p.(Met694Val) & c. $2080 \mathrm{~A}>\mathrm{G}$ & p.(Met694Val) & & hom & $A R, A D$ & 5 & \\
\hline 13 & MEFV & c. $2080 A>G$ & p.(Met694Val) & c. $2080 A>G$ & p.(Met694Val) & & hom & $A R, A D$ & 5 & \\
\hline 14 & MEFV & c. $2080 A>G$ & p.(Met694Val) & c. $2080 A>G$ & p.(Met694Val) & & hom & $A R, A D$ & 5 & \\
\hline 17 & MEFV & c. $2040 G>C$ & p.(Met680Ile) & c. $2040 \mathrm{G}>\mathrm{C}$ & p.(Met680lle) & & hom & $A R, A D$ & 5 & \\
\hline 4 & MEFV & c. $2040 \mathrm{G}>\mathrm{C}$ & p.(Met680lle) & c. $2282 \mathrm{G}>\mathrm{A}$ & p.(Arg761His) & & comp-het & $A R, A D$ & $5 / 4$ & \\
\hline 10 & MEFV & c. $2040 \mathrm{G}>\mathrm{C}$ & p.(Met680Ile) & c. $2282 \mathrm{G}>\mathrm{A}$ & p.(Arg761His) & & comp-het & $A R, A D$ & $5 / 4$ & \\
\hline 1 & MEFV & c. $2080 A>G$ & p.(Met694Val) & - & - & & het & $A R, A D$ & 5 & \\
\hline 11 & MEFV & c. $2080 A>G$ & p.(Met694Val) & - & - & & het & $A R, A D$ & 5 & \\
\hline 15 & MEFV & c. $2080 A>G$ & p.(Met694Val) & - & - & & het & $A R, A D$ & 5 & \\
\hline 31 & MEFV & c. $2082 A>G$ & p.(Met694lle) & - & - & & het & $A R, A D$ & 5 & \\
\hline 12 & MEFV & c. $2177 T>C$ & p.(Val726Ala) & - & - & & het & $A R, A D$ & 5 & \\
\hline 7 & MEFV & c. $2040 \mathrm{G}>\mathrm{C}$ & p.(Met680lle) & - & - & & het & $A R, A D$ & 5 & \\
\hline 8 & MEFV & c. $2040 \mathrm{G}>\mathrm{C}$ & p.(Met680lle) & - & - & & het & $A R, A D$ & 5 & \\
\hline 16 & MEFV & c. $2040 \mathrm{G}>\mathrm{C}$ & p.(Met680lle) & - & - & & het & $A R, A D$ & 5 & \\
\hline 125 & MEFV & c. $2040 G>C$ & p.(Met680Ile) & - & - & & het & $A R, A D$ & 5 & \\
\hline 62 & MEFV & c. $2177 T>C$ & p.(Val726Ala) & - & - & & het & $A R, A D$ & 5 & \\
\hline 22 & NLRP3 & c. $1043 C>T$ & p.(Thr348Met) & - & - & NM_001079821.2 & het & $A D$ & 5 & $\begin{array}{l}\text { CINCA syndrome (MIM607115) } \\
\text { Deafness, autosomal dominant 34, with } \\
\text { or without inflammation (MIM617772) } \\
\text { Familial cold inflammatory syndrome } 1 \\
\text { (MIM120100) }\end{array}$ \\
\hline
\end{tabular}




\begin{tabular}{|c|c|c|c|c|c|c|c|c|c|c|}
\hline & & & & & & & & & & $\begin{array}{l}\text { Keratoendotheliitis fugax hereditaria } \\
\text { (MIM148200) } \\
\text { Muckle-Wells syndrome (MIM191900) }\end{array}$ \\
\hline 19 & $A D A 2$ & c. $139 \mathrm{G}>\mathrm{A}$ & p.(Gly47Arg) & c. $139 \mathrm{G}>\mathrm{A}$ & p.(Gly47Arg) & NM_001282225.2 & hom & $A R$ & 5 & $\begin{array}{l}\text { Sneddon syndrome (MIM182410) } \\
\text { Vasculitis, autoinflammation, } \\
\text { immunodeficiency, and hematologic } \\
\text { defects syndrome (MIM615688) }\end{array}$ \\
\hline 23 & CYBB & c. $1511 \mathrm{G}>\mathrm{C}$ & p.(Gly504Ala) & - & - & NM_000397.3 & hemi & $X L R$ & 4 & $\begin{array}{c}\text { Chronic granulomatous disease } \\
\text { (MIM306400) } \\
\text { Immunodeficiency 34, mycobacteriosis } \\
\text { (MIM 300645) }\end{array}$ \\
\hline 18 & ILIORB & c. $477 \mathrm{G}>\mathrm{A}$ & p.(Trp159*) & c. $477 \mathrm{G}>\mathrm{A}$ & p.(Trp159*) & NM_000628.4 & hom & $A R$ & 4 & $\begin{array}{c}\text { Inflammatory bowel disease } 25 \\
\text { (MIM 612567) }\end{array}$ \\
\hline 55 & MS4A1 & c.203dup & p.(Leu69Serfs*72) & - & - & NM_152866.2 & het & $A R$ & 4 & $\begin{array}{l}\text { Immunodeficiency, common variable, } 5 \\
\text { (MIM613495) }\end{array}$ \\
\hline 25 & RIPK1 & c. $970 \mathrm{G}>\mathrm{C}$ & p.(Asp324His) & - & - & NM_001354930.1 & het & $A D$ & 5 & $\begin{array}{c}\text { Autoinflammation with episodic fever } \\
\text { and lymphadenopathy (MIM618852) } \\
\text { Immunodeficiency } 57 \text { with } \\
\text { autoinflammation (MM618108) }\end{array}$ \\
\hline 20 & TTC37 & c. $2453 \_2454$ delTG & p.(Val818Glufs*5) & $\begin{array}{c}\text { c. }(-228+1216-- \\
228+1222)_{-}^{-} \\
\left(326+92 \_326+98\right)\end{array}$ & p.? & NM_014639.3 & comp-het & $A R$ & $4 / 4$ & $\begin{array}{l}\text { Tricho-hepato-enteric syndrome } 1 \\
\text { (MIM222470) }\end{array}$ \\
\hline
\end{tabular}





\section{Figure Legends}

\section{Figure 1: Genetic workflow displaying step-by-step diagnostic strategy.}

First, WES data from all patients were screened using virtual VPANEL_1 (13 genes); in case of nonconfirmatory findings, virtual VPANEL_2 was analyzed (420 genes). In a third step, WES data (WES_3) were filtered to detect the most likely deleterious variants highly suspicious for AID. Additionally, HPO terms were used according to the patient's phenotype. If applicable, trio WES were performed; PV= pathogenic, or LPV= likely pathogenic variant found through genetic diagnostics.

\section{Figure 2: Final diagnosis of pediatric AID cohort after genetic testing.}

The pie chart is presenting the final diagnosis of 125 pediatric patients with AID categorized into the following subclasses: Genetic autoinflammation (AID), juvenile idiopathic arthritis (JIA), PFAPA, patients with transient symptoms, unclassified SAID, Mobus Behçet, other defined disease of the immune system (no genetic cause identified) and Inborn Errors of Immunity (IEI) (other than autoinflammation). AID was subdivided into FMF, VAIHS-syndrome, CAPS and CRIA syndrome. $n=$ amount of patients.

\section{Figure 3: Phenotypes in FMF}

Radar chart visualizing the symptoms of biallelic and monoallelic carriers of pathogenic MEFV variants depending on zygosity. [Turquoise line: amount of patients with monoallelic variants in \%, orange line: amount of patients with biallelic variants in \%.].

Figure 4: Serum levels of inflammatory biomarkers measured in patients with pathogenic MEFV variants. Each dot corresponds to a value in one patient; cross symbolizes the mean value of all patients; red line drawn at reference serum level cut-off point a) serum calprotectin levels of monoallelic and biallelic carriers of pathogenic MEFV variants; b) serum amyloid A levels of monoallelic and biallelic carriers of pathogenic MEFV variant. 


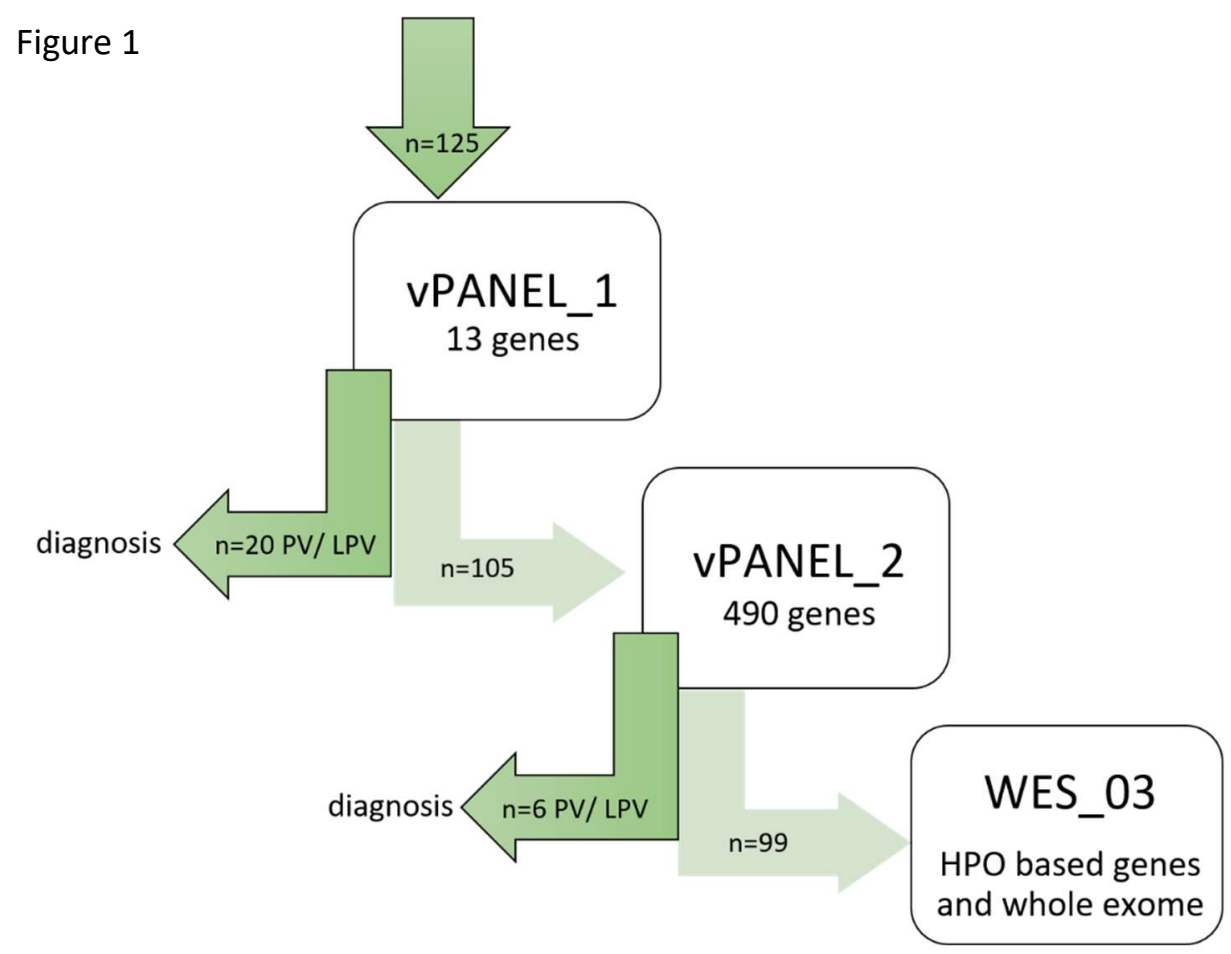

Figure 2

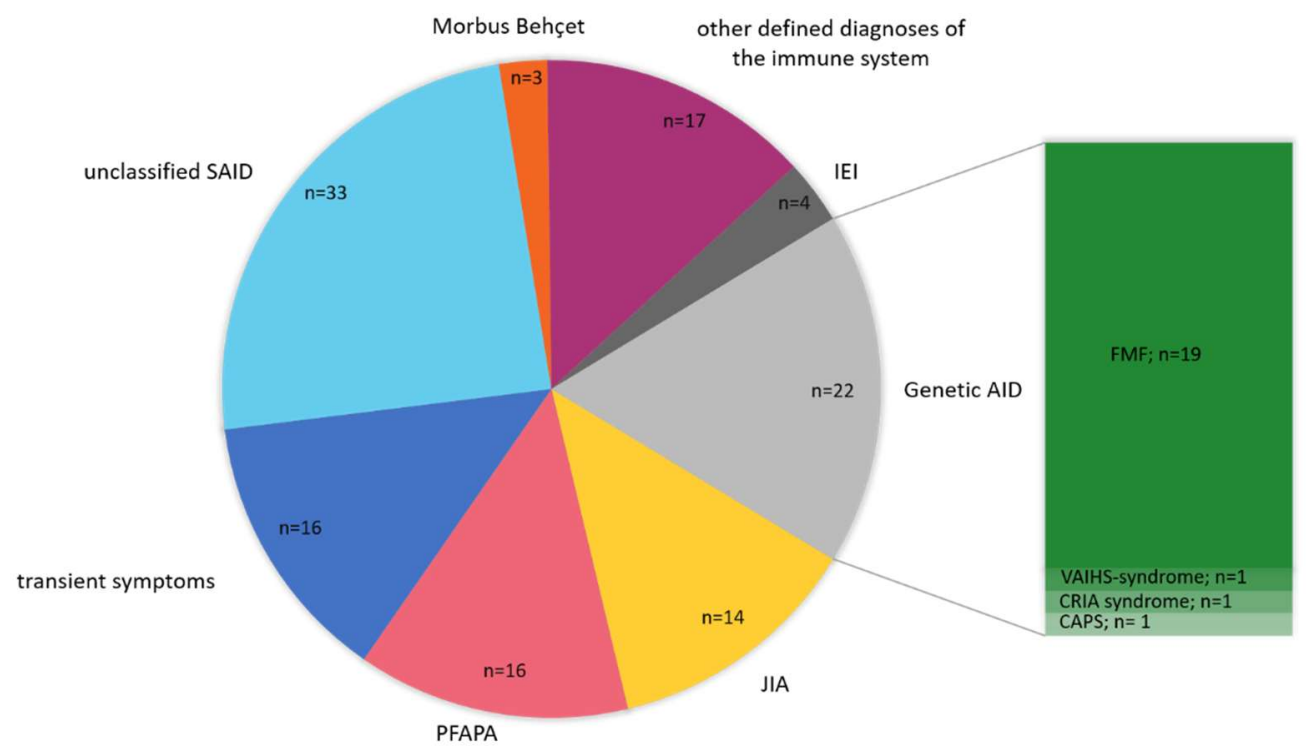


Figure 3

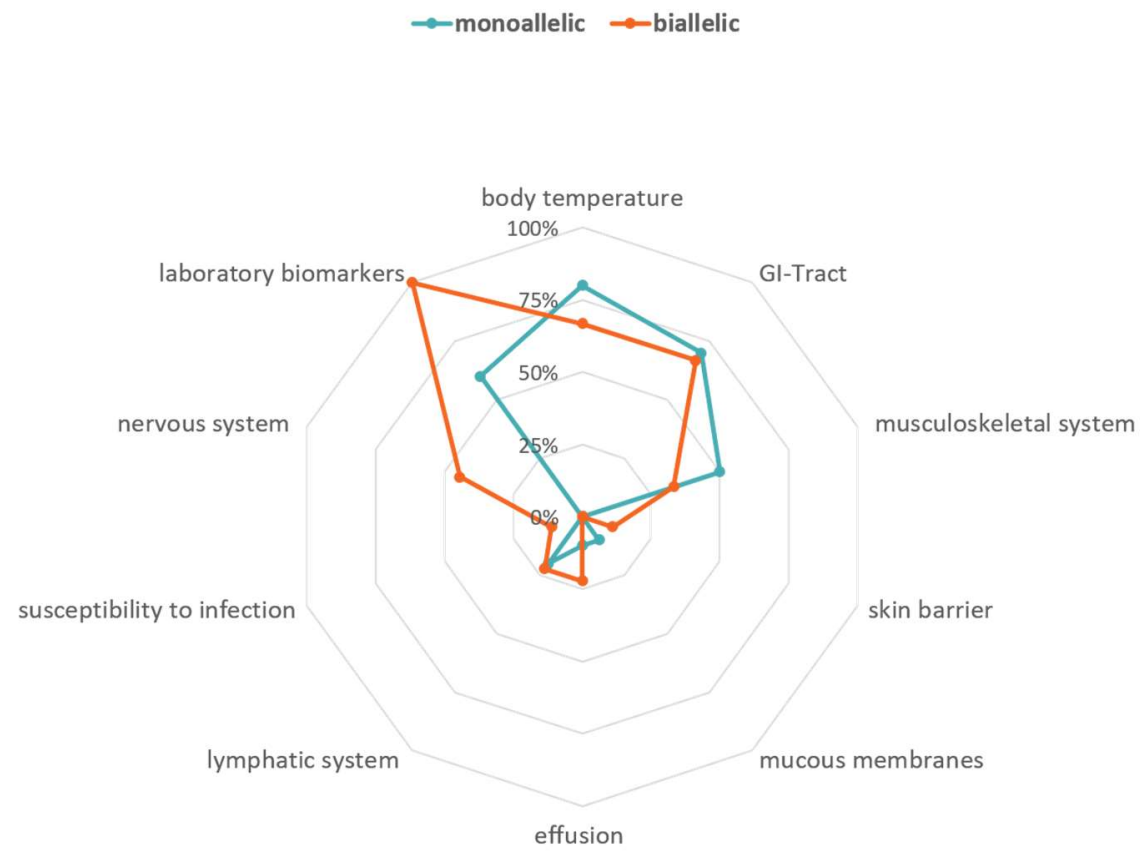

Figure 4
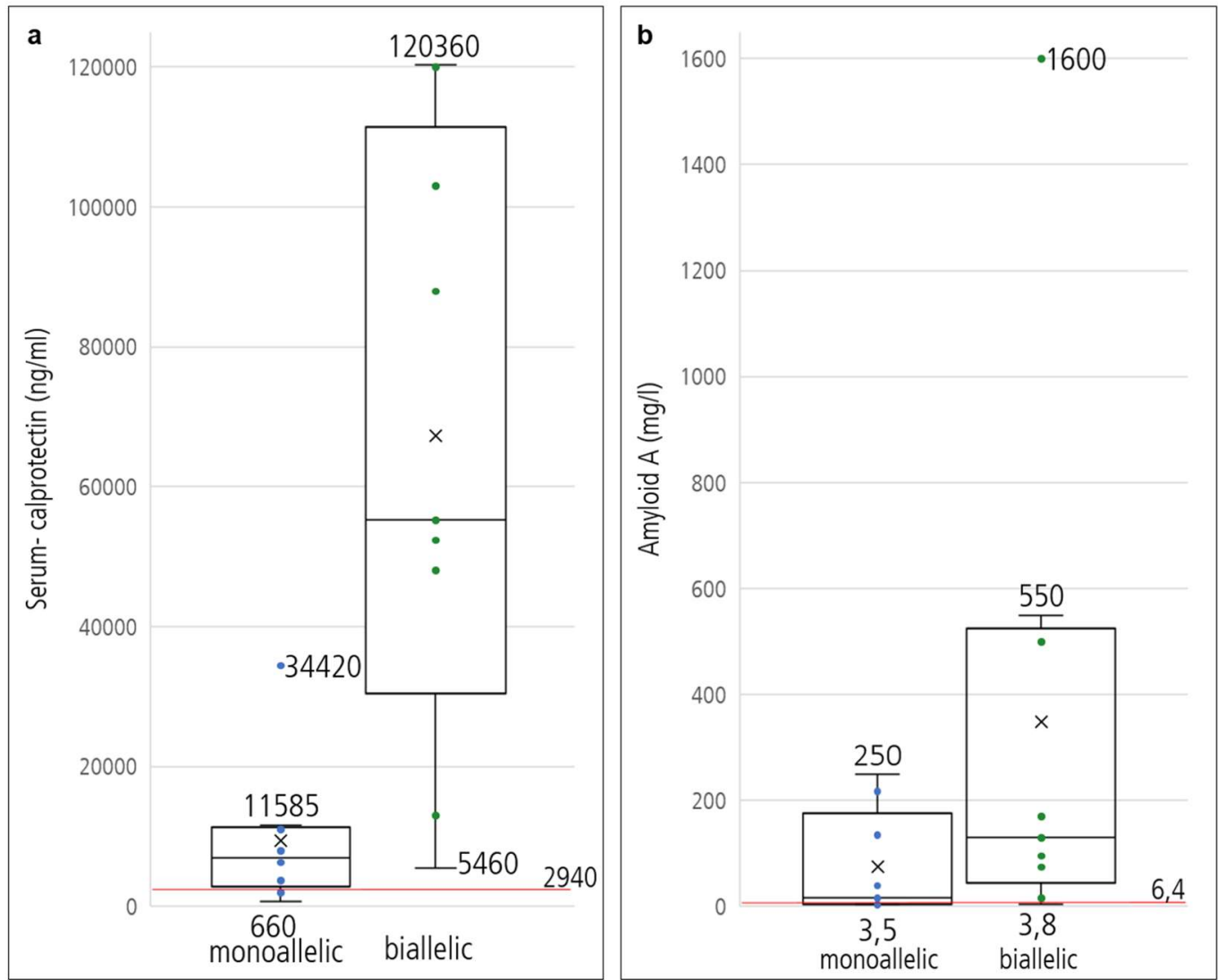


\section{Supplementary Files}

This is a list of supplementary files associated with this preprint. Click to download.

- JOCISupplementaldata.pdf

- JOCISupplementaltable.xlsx 